\title{
Craniofrontonasal dysplasia-Poland anomaly syndrome
}

INSERM

\section{Source}

INSERM. (1999). Orphanet: an online rare disease and orphan drug data base.

Craniofrontonasal dysplasia-Poland anomaly syndrome. ORPHA:1521

Cranio-fronto-nasal dysplasia - Poland anomaly is a polymalformative syndrome characterised by craniosynostosis, Poland anomaly (see this term), cranio-fronto-nasal dysplasia, and genital and breast anomalies. Less than ten cases have been described so far. 